\title{
Integration of Cryo-FIB-SEM Imaging into Dynamic Thermo-fluidic Experimentation: Applications to Multifunctional Nanoengineered Surface Design
}

\author{
K. Rykaczewski
}

\section{School for Engineering of Transport, Matter and Energy, Arizona State University, Tempe, AZ 85287}

Cryogenic FIB-SEM is typically applied for milling and imaging of samples that are static in nature prior to cryopreservation, e.g. cells [1], sunscreen [2] or hydrated clays [3]. In contrast, here new routes of integrating cryo-FIB-SEM imaging into dynamic thermo-fluidic experimentation outside the microscope are described. This approach enables cross-sectional and 3D nanoscale imaging of different stages of "macro" experiments (e.g. condensation, frost or ice-growth). To perform these experiments, cryo-FIB-SEM sample holders (i.e. stubs and "shuttles") are transformed of into experimental platforms compatible with rapid cryopreservation. Plunge-freezing in liquid nitrogen slush is used to "stop" the dynamic experiment at desired stages. From technical standpoint, this task often requires building of a setup that balances contradictory processes, for example, avoiding flooding of transfer rod-shuttle connection while condensing droplets on a sample. To directly correlate "macro" and "nano" imaging, an optical microscope is also incorporated into the experimental setup. Dependent on the specific problem, multiple processing steps after cryopreservation within liquid nitrogen or the transfer chamber can be performed to reveal the desired interface. Here three case studies that utilize variations of this approach to determine underlying nanoscale mechanisms of various surface phenomena occurring on nanotextured superhydrophobic (SHS) and hybrid solid-liquid omniphobic lubricant-impregnated surfaces (LIS) are briefly described.

Condensation rate enhancement on SHS and LIS: Application of both of these types of surfaces has been demonstrated to significantly increase water condensation rates [4-6], which could provide significant efficiency enhancement in power generation and desalination plants. However, nucleation and droplet dynamics stemming from the geometry of condensate-surface interface are not well understood. To study the morphology of this interface, a setup using custom thermocouple embedded sample stubs mounted onto a thermoelectric cooling stage in a high humidity conditions was developed [7]. As shown in Fig. 1a \& b, the setup also included an optical microscope and liquid nitrogen slushing chamber. Example cryo-FIB-SEM images of interface between lens-like drops on Krytox oil impregnated nanowire forest is shown in Fig. 1f, respectively.

Frost formation and ice-adhesion mechanisms on LIS: These coatings have also been reported to have extreme anti-frosting and anti-icing properties [8], which could have significant impact on aviation industry. However, integrated frosting and cryo-FIB-SEM imaging experiments revealed that the lubricant is drained into frost layer during dendritic nano-icicle growth (Fig. 1g) [9]. The lubricant draining process was shown to compromise the LIS anti-frosting functionality. In turn, imaging of LISbulk ice interface required development of two new sample handling approaches due to the required $\sim 3$ to $4 \mathrm{~mm}$ ice layer thickness. This thick ice layer was formed by freezing water at $-10^{\circ} \mathrm{C}$ inside of a temporary reservoir consisting of a $\sim 5 \mathrm{~mm}$ tall flexible tubing tightly fitted around a circular stub with mounted sample. Subsequently the ice-surface interface was revealed through either (1) mechanical break-up followed by sublimation of majority of the ice in the vacuum transfer chamber followed by FIB-cross sectioning (Fig. 1d, e \& h) or (2) flipping of the iced-over sample upside-down, fracturing-off the sample, and imaging its imprint in the ice-layer (Fig. 1i). The results revealing nanoscale defects in the LIS-ice interface, shown in images in Figure 1d \& e, proved to be crucial in explaining counterintuitive macroscale ice adhesion strength trends [10-11]. 


\section{References:}

[1 ] Hayles, M. F.; Stokes, D. J.; Phifer, D.; Findlay, K. C., J.Microsc. 226 (2007), p. 263.

[2] Scott, K., Microsc. Microanal. 17 (2011), p. 668.

[3] Desbois, G. et al. Geofluids 8 (2008), p. 60.

[4] Dietz, C.; Rykaczewski, K.; Fedorov, A. G.; Joshi, Y., App. Phys. Lett. 97 (2010), p. 033104.

[5] Rykaczewski, K. et al. Soft Matter 8 (2012), p. 8786.

[6] Anand, S. et al. ACS Nano 6 (2012), p. 10122.

[7] Rykaczewski, K. et al. ACS Nano 6 (2012), p. 9326.

[8] Kim, P. et al. ACS Nano 6 (2012), p. 6569.

[9] Rykaczewski, K. et al. Langmuir 29 (2013), p. 5230.

[10] Bengaluru Subramanyam, S.; Rykaczewski, K.; Varanasi, K. K., Langmuir 2013.

[11] Funding from Fulton Schools of Engineering at ASU and NIST for use of FIB-SEM facilities. Part of the described worked was carried out at ASU's LeRoy Eyring Center for Solid State Science. Contact: konradr@asu.edu
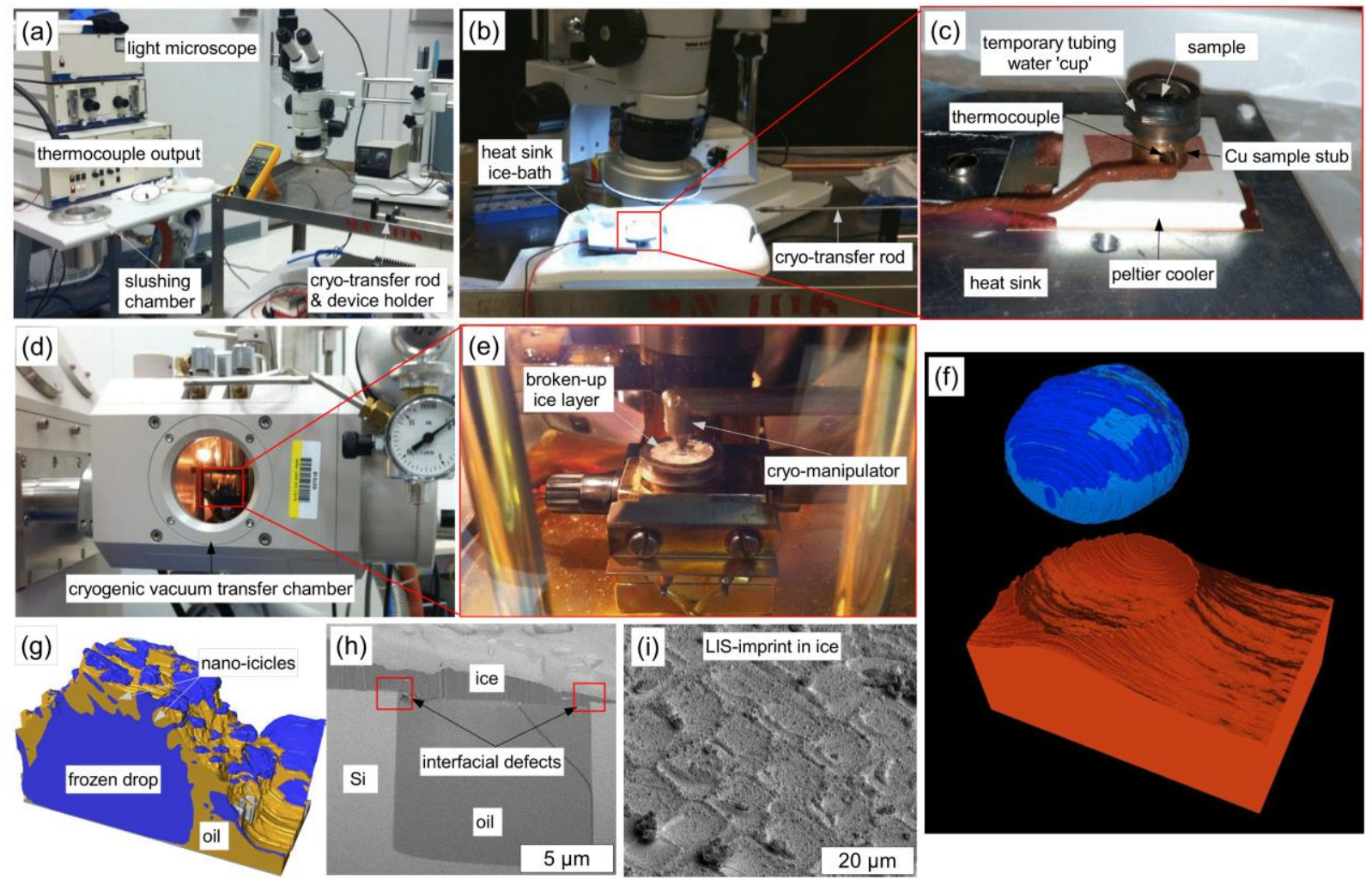

Figure 1. (a-c) Condensation, frosting, and icing setup with liquid nitrogen slushing and vacuum cryotransfer chambers, (b) example of icing experiment with optical recording of the process, (c) details of the sample holder with temporary water "cup" consisting of flexible tubing around the sample stub, (d) $\&$ (e) breaking-up and sublimation of the thick ice layer to expose the ice-LIS interface, (f-i) example cryo-FIB-SEM results of (f) droplet condensed on LIS (drop is lifted from the substrate to reveal the shape of the oil meniscus) [7], (g) frozen drop with nano-icicles flooded by oil [9], (h) cross-section of ice-LIS interface showing interfacial defects, and (i) corresponding imprint of LIS in the ice [10]. 